\title{
Supercapsular percutaneously-assisted total hip (SuperPath) versus mini-incision posterolateral total hip arthroplasty for hip osteoarthritis: a prospective randomized controlled trial
}

\author{
Weikun Meng ${ }^{1,2,3 \#}$, Liang Gao ${ }^{2,3 \#}{ }^{1}$, Zhong Huang ${ }^{1,3}$, Haoyang Wang ${ }^{1}$, Duan Wang ${ }^{1}$, Zeyu Luo ${ }^{1}$, Yang Bai ${ }^{4}$, \\ Guanglin Wang ${ }^{1}$, Zongke Zhou ${ }^{1}$ \\ ${ }^{1}$ Department of Orthopaedics, West China Hospital, West China School of Medicine, Sichuan University, Chengdu, China; ${ }^{2}$ Center of Experimental \\ Orthopaedics, Saarland University Medical Center, Homburg/Saar, Germany; ${ }^{3}$ Sino Euro Orthopaedics Network (SEON), Homburg/Saar, \\ Germany; ${ }^{4}$ Department of Immunization, Yunnan Center for Disease Control and Prevention, Kunming, China \\ Contributions: (I) Conception and design: W Meng, Z Zhou; (II) Administrative support: H Wang, L Gao, G Wang, Z Zhou; (III) Provision of \\ study materials or patients: W Meng; (IV) Collection and assembly of data: L Gao, Z Huang, H Wang, D Wang, Z Luo; (V) Data analysis and \\ interpretation: L Gao, Z Huang, Y Bai; (VI) Manuscript writing: All authors; (VII) Final approval of manuscript: All authors. \\ \#These authors contributed equally to this work. \\ Correspondence to: Liang Gao. Center of Experimental Orthopaedics, Saarland University, Kirrberger Strasse, Building 37, D-66421 Homburg/Saar, \\ Germany. Email: lianggao@web.de; Zongke Zhou. Department of Orthopaedics, West China Hospital, Sichuan University, No. 37, Wuhou Guoxue \\ Road, Chengdu 610041, China. Email: zongke@126.com.
}

Background: Supercapsular percutaneously-assisted total hip (SuperPath) arthroplasty has been proposed to be minimally invasive and tissue sparing, with possible superior postoperative outcomes compared with conventional approaches for total hip arthroplasty (THA). However, previous studies have underlined the shortcomings of conventional THA approaches, including higher dislocation, more blood loss, longer incisions, more tissue damage, and delayed postoperative rehabilitation. In the present study, we compared the short-term outcomes of unilateral THA with those of SuperPath and the mini-incision posterolateral approach (PLA) for hip osteoarthritis (OA).

Methods: Patients with unilateral hip OA were prospectively recruited and underwent either SuperPath (SuperPath group) or mini-incision PLA THA (PLA group). Perioperative status [operative time, incision length, intraoperative blood loss, soft tissue damage, and length of hospital stay (LOS)], and postoperative function outcomes, including range of motion (ROM), pain visual analog scale (VAS), and Harris Hip Score (HHS), were evaluated and compared between the groups at scheduled time points within 12 months postoperatively.

Results: Compared with the PLA group, the SuperPath group yielded a significantly shorter incision length (7.83 vs. $12.45 \mathrm{~cm}, \mathrm{P}<0.001)$, longer operative time (102.72 vs. $66.22 \mathrm{~min}, \mathrm{P}<0.001)$, more blood loss (1,007.38 vs. $844.55 \mathrm{~mL}, \mathrm{P}=0.005)$, and more soft tissue damage (creatine kinase: 1,056.05 vs. $821.50 \mathrm{U} / \mathrm{L}$, $\mathrm{P}=0.006$ ) on postoperative day 3 . The SuperPath group also showed deficient acetabular cup positioning (abduction angle: $36.94^{\circ}$ vs. $42.66^{\circ}, \mathrm{P}=0.004$ ) and a greater decrease in $\mathrm{ROM}$ (flexion: $107.66^{\circ}$ vs. $114.44^{\circ}$, $\mathrm{P}=0.004 ; 109.83^{\circ}$ vs. $116.11^{\circ}, \mathrm{P}=0.002 ; 111.66^{\circ}$ vs. $\left.118.88^{\circ}, \mathrm{P}<0.001\right)$ on postoperative days 1,3 , and 14 , as well as severe early-term pain symptoms (pain VAS on postoperative day 3: 7.05 vs. 6.55, $\mathrm{P}=0.041$ ). However, the LOS, C-reactive protein levels, erythrocyte sedimentation rate (within 2 weeks postoperatively), and HHS were comparable between the groups during the 12 months postoperatively.

Conclusions: SuperPath may be a promising, minimally invasive technique for the treatment of OA in the future. Further investigation is necessary to evidence the possible superiority of SuperPath over other conventional mini-incision THA approaches.

^ ORCID: 0000-0003-1438-9670. 
Keywords: Minimally invasive surgery; hip osteoarthritis (hip OA); posterolateral approach (PLA); supercapsular percutaneously-assisted total hip arthroplasty (SuperPath arthroplasty); total hip arthroplasty (THA)

Submitted Feb 20, 2020. Accepted for publication Oct 29, 2020.

doi: $10.21037 / \mathrm{atm}-20-1793$ a

View this article at: http://dx.doi.org/10.21037/atm-20-1793a

\section{Introduction}

Osteoarthritis (OA) is the most common form of arthritis, and mainly affects the elderly $(1,2)$. It has been associated with heavy physical occupational activities $(3,4)$. If left untreated, hip OA typically leads to chronic pain, decreased quality of life, and various disabilities, and will often require total hip arthroplasty (THA) (5-8). As the global population ages, THA has become a high-volume surgical intervention for patients with end-stage hip OA $(9,10)$.

The conventional posterolateral approach (PLA) is the most widely utilized approach for THA, with excellent exposure for both primary and revision THA $(11,12)$. Postoperative dislocation, one of the most common postoperative complications, has been associated with traditional approaches of THA, and is possibly attributable to the intraoperative extensive impairment of periarticular soft tissue, especially the external rotators and joint capsules (13-15).

Recently, supercapsular percutaneously-assisted total hip (SuperPath) arthroplasty has emerged as a minimally invasive and tissue-sparing alternative to conventional THA approaches (16). In contrast to conventional approaches, this superior portal-assisted approach is proposed to access the hip capsule through the interval between the gluteus medius and piriformis and to preserve the periarticular soft tissues $(17,18)$, potentially yielding less postoperative dislocation and pain, better early function, and earlier hospital discharge $(16,19,20)$. However, in our recent study, which compared the 1-year outcomes between SuperPath and PLA THA for bilateral osteonecrosis of the femoral head, SuperPath was associated with longer operative time, more intraoperative blood loss, deficient abduction angle of the acetabular cup, and inferior early-term hip function within 12 months postoperatively (21). Despite the similar cumulative utilization of SuperPath for patients with OA, the evidence is insufficient to support its clinical benefits over other conventional approaches to THA.

The present study aimed to compare the short-term outcomes between SuperPath and PLA THA for unilateral end-stage primary OA patients. We hypothesized that
SuperPath would yield superior outcomes for OA patients compared to PLA THA, with better perioperative status [operative time, incision length, intraoperative blood loss, soft tissue damage, and length of hospital stay (LOS)] and improved postoperative function [range of motion (ROM), pain, and hip function]. We present the following article in accordance with the CONSORT reporting checklist (available at http://dx.doi.org/10.21037/atm-20-1793a).

\section{Methods}

\section{Patient selection}

Patients with unilateral primary hip OA were recruited from our department between March 2017 and June 2018. The study was conducted according to the principles of the Declaration of Helsinki (as revised in 2013). This study was approved by the Institutional Review Board of West China Hospital, West China School of Medicine, Sichuan University (No. 2016005). and registered on the Research Registry (https://www.researchregistry.com; No. Researchregistry5326). Patient characteristics [age, sex, body mass index (BMI), comorbidities, and American Society of Anesthesiologists (ASA) grade] were collected to determine whether the two cohorts were comparable at baseline. The present study enrolled patients with unilateral end-stage primary hip OA. All patients provided signed consent for implanting and agreed to complete the scheduled postoperative 12-month followup. Patients were excluded if they had non-inflammatory degenerative joint diseases (e.g., osteonecrosis, bilateral OA, and post-traumatic arthritis), inflammatory joint diseases (e.g., reactive arthritis, ankylosing spondylitis, rheumatoid arthritis, and gout), inadequate neuromuscular status (e.g., previous paralysis and inadequate abductor strength), and overt infections or distant foci of infections.

\section{Surgical approach}

All surgical procedures were completed by the same team, led by a senior surgeon (Z Zhou) specializing in lower limb 
reconstruction, with over 15 years' experience performing primary and revision THA (over 250 cases annually). To minimize the influence of a learning curve, the senior surgeon had performed $>50$ SuperPath cases prior to the present trial. SuperPath and mini-incision PLA THA were performed with specific prostheses (SuperPath group: Microport Orthopaedics, Arlington, TN, USA; PLA group: DePuy Synthes, Warsaw, IN, USA), as previously described $(11,22)$. Both approaches were randomized for each patient, using a shuffled deck of cards (even: SuperPath, odd: PLA) and were performed in either the right or left hip. This was a doubleblind study, in which the specific approach type was unknown to both the patients and the examiners (Z Huang, D Wang, Y Bai) who assessed patient outcomes.

Preoperative and postoperative data for each patient, including the age, sex, etiology, age of pain onset, history of hip injury/surgery, BMI, comorbidities (e.g., chronic obstructive pulmonary disease, congestive heart failure, diabetes, and hypertension), and ASA grade, were collected by 3 residents ( $Z$ Huang, H Wang, and Z Luo) (23). Operative time was defined as the total duration from skin incision to closure of the wound (D Wang), and incision length was approximated with linen tape along the surgical incision. The LOS, transfusion rate, and postoperative complications were also recorded, along with hospital readmission within 1 year after surgery. Standardized postoperative care was provided, including infection prophylaxis, venous thromboembolism prevention, nausea and vomiting management, wound care, and functional rehabilitation.

\section{Pain management}

Multi-modal pain management was offered to all patients as part of standardized postoperative care (24). Patients received preoperative femoral nerve block and prophylactic oral celecoxib $(200 \mathrm{mg})$. An intraoperative periarticular cocktail injection $(100 \mathrm{~mL})$ was injected into the surgical site with $0.5 \%$ ropivacaine $(49.25 \mathrm{~mL}), 0.5 \mathrm{mg}$ epinephrine $(0.5 \mathrm{~mL})$, $30 \mathrm{mg}$ ketorolac $(1 \mathrm{~mL}), 80 \mathrm{mcg}$ clonidine $(0.8 \mathrm{~mL})$, and sterile water $(48.45 \mathrm{~mL})$. Postoperatively, an intravenous patient-controlled morphine pump and oral celecoxib were administered for pain control, depending on pain intensity. After discharge, oral celecoxib or other non-steroidal antiinflammatory drugs were used as appropriate.

\section{Postoperative rehabilitation}

Both groups of patients undertook an identical rehabilitation program, which was delivered by an experienced physical therapist who received extensive training in managing postoperative hip conditions before this study. The physical therapist was blinded to the patient allocation. Briefly, immediate hip flexion, pneumatic compression with foot pumps, and deep breathing exercises were emphasized to minimize thromboembolic and pulmonary complications (25). After obtaining written approval from the physical therapist, patients began indoor walking independently with a tolerated weight bearing. Patients were educated in self-care and homebased rehabilitation before discharge. They were instructed to walk daily and to gradually increase their walking distance toward a goal of $2 \mathrm{~km} /$ day. All patients were discharged on postoperative day 3 and allowed to walk with a cane.

\section{Perioperative total blood loss}

The total blood loss was calculated by applying the Gross and Nadler formulas, as previously described (21).

\section{Perioperative serum markers}

To assess intraoperative soft tissue damage, serum markers, including creatine kinase $(\mathrm{CK}), \mathrm{C}$-reactive protein (CRP), and the erythrocyte sedimentation rate (ESR), were measured for each patient before surgery and on postoperative days 1,3 , and 14 (26).

\section{Acetabular component positioning analysis}

Standardized anteroposterior pelvic radiographs were acquired on both the day of hospital admission and postoperative day 1 . Inclination and anteversion angles of the acetabular component for each patient were measured with a computer-assisted measurement system (Japan Medical Material, Osaka, Japan), as previously described (27).

\section{Pain, ROM, and bip function}

The pain visual analog scale (VAS) measures pain on a scale from 0 (no pain) to 10 (worst imaginable pain). The VAS was used to measure pain on the day of hospital admission, on postoperative days 1,3 , and 14 , and at 3, 6, and 12 months after surgery (28). ROMs were measured using a goniometer the day before surgery, and at 3,6, and 12 months postoperatively (29). Harris Hip Score (HHS) was determined on the day of hospital admission, on postoperative days 1,3 , and 14 , and at 3,6 , and 12 months 


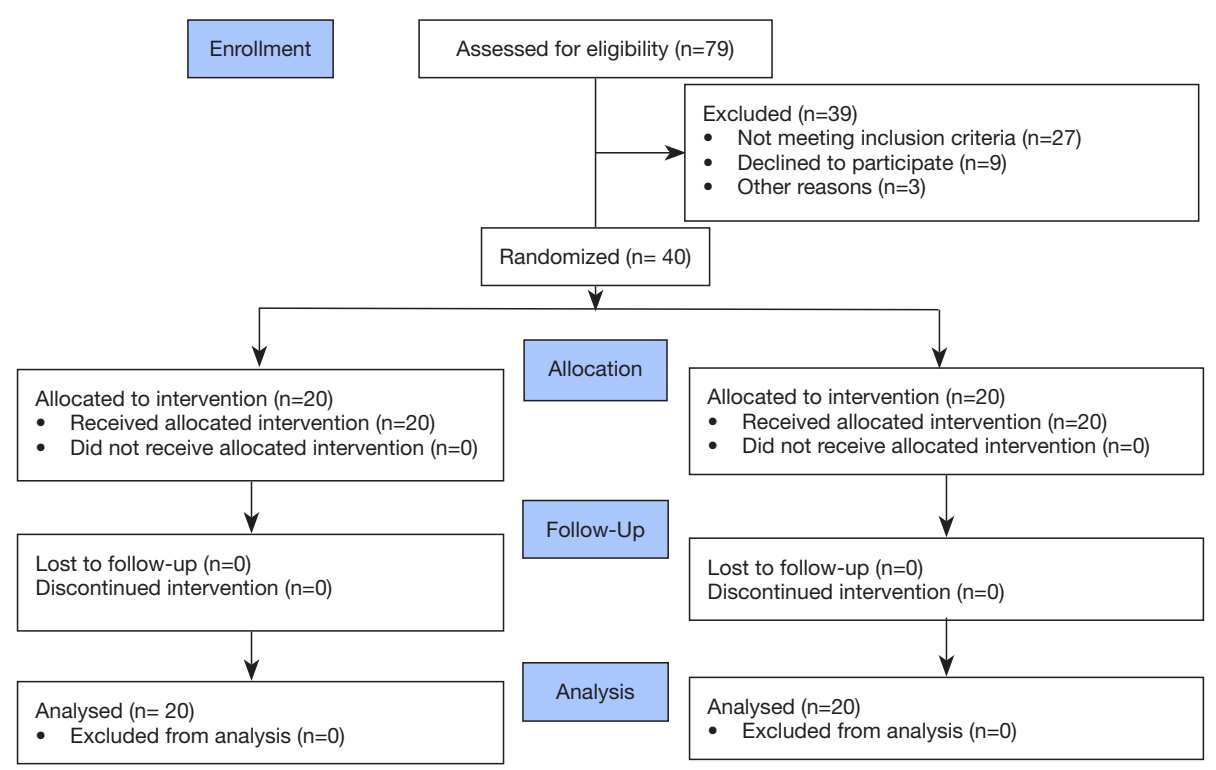

Figure 1 CONSORT flow diagram of patient recruitment and follow-up.

post-surgery (30).

\section{Statistical analysis}

Values were expressed as means \pm standard deviations, unless otherwise stated. Analysis of variance (ANOVA) was used to compare age and BMI between the groups, and the $\chi^{2}$ test was used to compare sex, ASA grade, comorbidities, and the transfusion rate. Continuous data were analyzed with the Student's $t$-test or non-parametric Mann-Whitney U-test as appropriate. One-way ANOVA was performed to compare the VAS and HHS between the different assessment time points. $\mathrm{P}<0.05$ was considered statistically significant. Calculations were performed using SPSS version 24 (SPSS Inc., Chicago, IL, USA).

\section{Results}

\section{Patient demographics and surgical details}

Forty patients were enrolled in the present study and randomized evenly into the SuperPath and PLA groups (Figure 1). All patient demographic characteristics were compared between the groups, including age, sex, BMI, comorbidities, and ASA grade (Table 1).

The incision length in the SuperPath group was significantly shorter than that in the PLA group (Table 1). However, the operative time was significantly longer in the SuperPath group than in the PLA group. Similarly, the mean blood loss was significantly higher in the SuperPath group. However, both groups were comparable in terms of the LOS, blood transfusion rate, postoperative complications, and hospital re-admission/reoperation rate within 12 months postoperatively.

\section{Perioperative serum markers change}

The serum markers (CK, CRP, and ESR) showed equivalent trends in both groups within the 2 weeks postoperatively (Figure 2 and Table 2). CK and CRP levels in the SuperPath group were higher than those in the PLA group at all assessment time points. Specifically, the CK level in the SuperPath group $(1,056.05 \pm 232.05 \mathrm{mg} / \mathrm{L})$ was higher than that of the PLA group on postoperative day $3(821.50 \pm 187.98 \mathrm{mg} / \mathrm{L}, \mathrm{P}=0.006)$, and the difference was significant. All serum markers reached maximal levels (CK: 1,056.05 vs. $821.50 \mathrm{mg} / \mathrm{L}, \mathrm{CRP}: 78.18$ vs. $61.20 \mathrm{U} / \mathrm{L}$, and ESR: 44.00 vs. $46.05 \mathrm{~mm} / \mathrm{h}$ in the SuperPath and PLA groups, respectively) on postoperative day 3 , and were comparable on postoperative day 14 .

\section{Acetabular cup position}

Postoperative radiographs showed that the cup abduction angle in the SuperPath group $\left(36.94^{\circ}\right.$ ) was significantly lower than that in the PLA group $\left(42.66^{\circ}, \mathrm{P}=0.004\right)$ (Table 3). 
Table 1 Demographic characteristics

\begin{tabular}{|c|c|c|c|}
\hline Characteristics & SuperPath $(n=20)$ & PLA $(n=20)$ & $P$ value \\
\hline Sex, male/female & $8 / 12$ & $9 / 11$ & 0.749 \\
\hline Mean BMI (SD), kg/m² & $23.36(2.55)$ & $22.82(2.61)$ & 0.414 \\
\hline \multicolumn{4}{|l|}{ Diabetes, n [\%] } \\
\hline Type II & 7 [35] & $9[45]$ & 0.519 \\
\hline Smoking, $\mathrm{n}[\%]$ & $11[55]$ & 9 [45] & 0.527 \\
\hline COPD, n [\%] & $2[10]$ & $3[15]$ & 0.633 \\
\hline $\mathrm{CHF}, \mathrm{n}[\%]$ & 0 & 0 & n.a. \\
\hline History of hip injury, n [\%] & 0 & 0 & n.a. \\
\hline History of hip surgery, n [\%] & 0 & 0 & n.a. \\
\hline ASA grade, $n[\%]$ & & & 1.000 \\
\hline 1 & 0 & 0 & \\
\hline 2 & 13 [65] & 13 [65] & \\
\hline 3 & $7[35]$ & $7[35]$ & \\
\hline 4 & 0 & 0 & \\
\hline \multicolumn{4}{|l|}{ Perioperative data, mean (SD) } \\
\hline Postoperative complications, $\mathrm{n}$ [\%] & 0 & 0 & n.a. \\
\hline Readmission within 12 months postoperatively, $\mathrm{n}$ [\%] & 0 & 0 & n.a. \\
\hline Reoperation within 12 months postoperatively, $\mathrm{n}$ [\%] & 0 & 0 & n.a. \\
\hline
\end{tabular}

Statistical analyses were performed with Mann-Whitney U-test or $\chi^{2}$ test, as appropriate. ${ }^{*}, \mathrm{P}<0.05$. SuperPath, supercapsular percutaneously-assisted total hip; PLA, posterolateral approach; SD, standard deviation; BMI, body mass index; COPD, chronic obstructive pulmonary disease; n.a., not applicable; CHF, congestive heart failure; ASA, American Society of Anesthesiologist.

The average cup anteversion angle between the groups was comparable.

\section{$R O M$}

ROM of the affected hip in the SuperPath group was significantly lower than that in the PLA group on postoperative days 1,3 , and 14 (SuperPath, $107.66^{\circ}$, $109.83^{\circ}, 111.66^{\circ}$; PLA, $114.44^{\circ}, 116.11^{\circ}, 118.88^{\circ}$; all $\mathrm{P}<0.05$ ) (Table 4). These significant differences had disappeared 3 months after surgery. However, abduction and external rotation ROMs remained similar between the groups at all assessment time points within 12 months postoperatively $(\mathrm{P}<0.815$ and $\mathrm{P}<0.696$, respectively). 

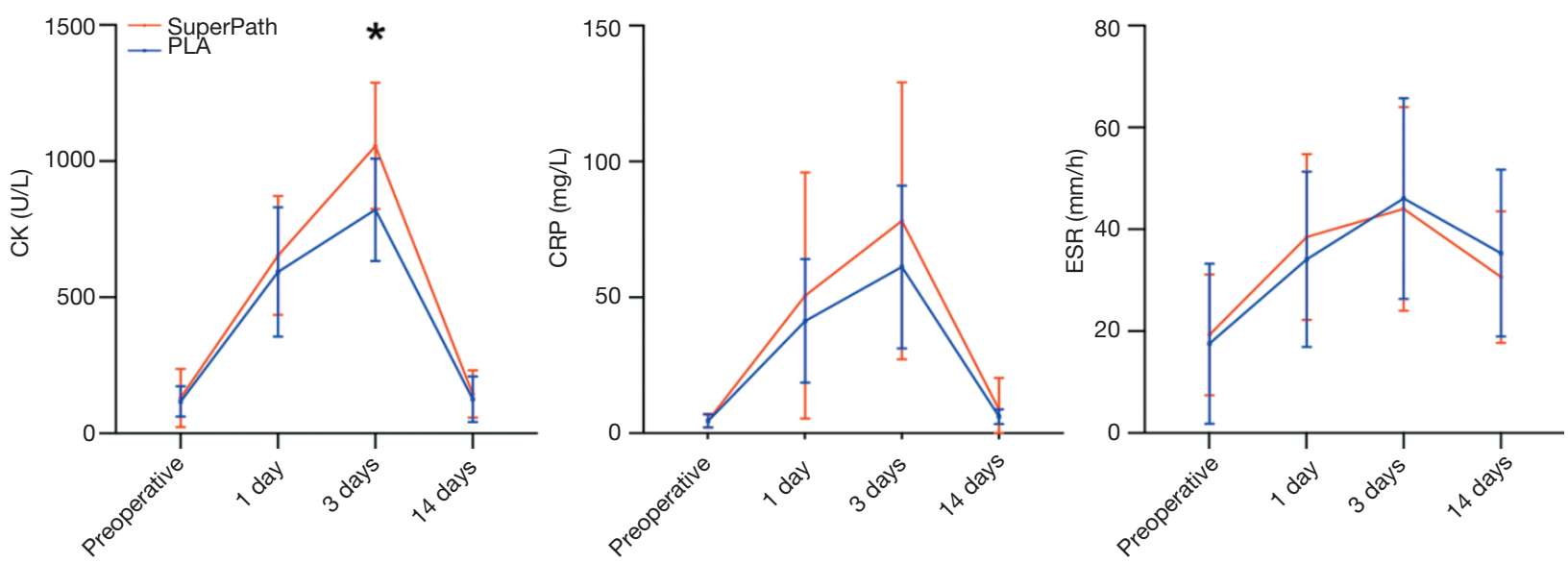

Figure 2 Perioperative changes of serum markers, including creatine kinase (CK), C-reactive protein (CRP), and the erythrocyte sedimentation rate (ESR), after unilateral total hip arthroplasty with either the supercapsular percutaneously-assisted total hip (SuperPath) or mini-incision posterolateral approach (PLA) for hip osteoarthritis. * $\mathrm{P}<0.05$ indicates statistical significance between the SuperPath group and PLA groups.

Table 2 Perioperative changes of serum markers

\begin{tabular}{lcccc}
\hline Variable & Time point & SuperPath $(\mathrm{n}=20)$ & PLA $(\mathrm{n}=20)$ & P value \\
\hline $\mathrm{CK}, \mathrm{U} / \mathrm{L}$ & Preoperative & $130.16(107.20)$ & $117.33(55.82)$ & 0.988 \\
& Postoperative day 1 & $653.66(218.23)$ & $592.88(237.34)$ & 0.424 \\
& Postoperative day 3 & $1,056.05(232.05)$ & $821.50(187.98)$ & $0.006^{*}$ \\
& Postoperative day 14 & $144.94(87.28)$ & $125.33(83.87)$ & 0.501 \\
CRP, mg/L & Preoperative & $4.65(2.56)$ & $4.50(2.36)$ & 0.938 \\
& Postoperative day 1 & $50.69(45.25)$ & $41.34(22.76)$ & 0.696 \\
& Postoperative day 3 & $78.18(50.94)$ & $61.20(29.98)$ & 0.462 \\
ESR, mm/h & Postoperative day 14 & $8.79(11.59)$ & $6.13(2.66)$ & 0.888 \\
& Preoperative & $19.27(11.84)$ & $17.55(15.69)$ & 0.252 \\
& Postoperative day 1 & $38.50(16.29)$ & $34.11(17.19)$ & 0.424 \\
& Postoperative day 3 & $44.00(20.00)$ & $46.05(19.71)$ & $35.33(16.38)$ \\
\hline
\end{tabular}

Data are reported as mean (standard deviation). Statistical analyses were performed with either Mann-Whitney U-test or $\chi^{2}$ test, where appropriate. * $\mathrm{P}<0.05$. SuperPath, supercapsular percutaneously-assisted total hip; PLA, posterolateral approach; CK, creatine kinase; CRP, C-reactive protein; ESR, erythrocyte sedimentation rate; SD, standard deviation.

\section{Pain and bip function}

On postoperative day 3, pain VAS was significantly higher in the SuperPath group (7.05) compared with the PLA group (6.55, $\mathrm{P}=0.041)$ and remained comparable between both groups at other time points within 1 year postoperatively (Figure 3 and Table 5). Interestingly, pain symptoms were significantly relieved on postoperative day $3(6.55 \pm 0.70)$ in the PLA group, but the SuperPath group did not show significant relief of pain symptoms until postoperative day $14(5.11 \pm 1.02)$ (Table 5). In both groups, the pain VAS reached its minimum plateau between 6 and 12 months postoperatively. 
Table 3 Radiological evaluation of acetabular cup positioning on postoperative day 1

\begin{tabular}{lccc}
\hline Variable & $\begin{array}{c}\text { SuperPath } \\
(\mathrm{n}=20)\end{array}$ & PLA $(\mathrm{n}=20)$ & P value \\
\hline Abduction angle $\left(^{\circ}\right)$ & $36.94(6.37)$ & $42.66(3.58)$ & $0.004^{*}$ \\
Anteversion angle $\left(^{\circ}\right)$ & $13.94(4.73)$ & $15.11(4.06)$ & 0.501 \\
\hline
\end{tabular}

Data are reported as mean (standard deviation). Statistical analyses were performed with either Mann-Whitney U-test or $\chi^{2}$ test, where appropriate. ${ }^{*}, \mathrm{P}<0.05$. PLA, posterolateral approach; SuperPath, supercapsular percutaneously-assisted total hip.

Differences in average HHS were not significant between the groups at any time point (Table 5). Further individual analyses of the HHS change pattern showed that hip function showed immediate improvement on postoperative day $1(\mathrm{P}<0.001)$ in both groups compared to preoperative baseline data (Table 6). Of note, hip function was observed to be significantly improved on postoperative day 14 (70.66 \pm 6.22$)$ in the PLA group, but only showed a significant improvement at 3 months postoperatively $(82.44 \pm 3.51)$ in the SuperPath group. Improvement in hip function reached its maximum plateau between 6 and 12 months in both groups ( 6 vs. 12 months postoperatively, $\mathrm{P}>0.05$ ).

\section{Discussion}

The present study demonstrated that, compared with the conventional PLA for unilateral THA for hip OA, SuperPath is associated with a significantly longer operative time, more total blood loss and soft tissue damage, deficient acetabular cup positioning, and inferior earlyphase postoperative recovery in terms of pain symptoms, ROM, and hip function within 12 months postoperatively. However, the incision length was considerably shorter with SuperPath, and the two groups had comparable outcomes for LOS and 1-year hip function.

Over past decades, various minimally invasive procedures have been proposed for THA, with the primary aims of minimizing soft tissue damage and improving postoperative cosmesis (31). The concept of surgical invasiveness cannot be limited to the length or size of the skin incision; it must be extended to all anatomical structures dissected during the procedure and essential issues relating to the entire perioperative phase, such as the type of anesthetic, operative time, intraoperative bleeding, postoperative pain, complication and success rates, and long-term outcomes (21).
To the best of our knowledge, this is the first study to show the SuperPath approach to have less favorable shortterm outcomes than conventional PLA THA for hip OA. SuperPath was found to have a significantly shorter incision length; however, it had a significantly longer operative time and more intraoperative blood loss than conventional PLA THA, even when performed by an experienced team. Of note, the SuperPath group unexpectedly yielded more soft tissue damage and significantly increased serum CK levels compared with the PLA group on day 3 postoperatively. These data are in accordance with a recent study that highlighted that the SuperPath technique resulted in significantly increased hidden blood loss compared with the conventional posterior approach for hip hemiarthroplasty (32). However, the findings of the present study were not in agreement with a previous case series that claimed that SuperPath was a tissue-sparing minimally invasive alternative to PLA THA that could intraoperatively preserve the periarticular soft tissue $(16,19,20,33-35)$. These inconsistent results are possibly attributable to additional intraoperative soft tissue (mainly muscle) damage from many sources (36), including intraoperative stretching (37), unintentional detachment (37), and varied instrument retraction $(38,39)$, during the significantly elongated time taken to perform the SuperPath procedure $(32,40-42)$. Therefore, more studies are needed to identify the factors that contribute to soft tissue damage during the SuperPath procedure and to determine the possible advantages of this approach over traditional techniques.

The significantly restricted hip ROM on postoperative day 3 and considerably increased pain symptoms within 2 weeks postoperatively were also observed in the SuperPath group compared with the PLA group. A higher serum CK level was also found in SuperPath group. Interestingly, both serum CK level and hip ROM were comparable between the groups after postoperative day 14 . This temporary aggravated ROM restriction in the SuperPath group might be attributable to both intraoperative soft tissue damage and postoperative pain. As stated by other authors, the clinical importance of postoperative CK values and associations between hip function with other clinical outcomes (e.g., soft tissue damage, pain symptoms, and blood loss) warrants further study $(21,32)$.

Although perioperative pain VAS and HHS at various time points were generally comparable, chronological comparisons of these data revealed these that these two parameters showed different patterns between the groups. Pain VAS significantly improved as early as postoperative day 3 in the PLA group, while the improvement in the SuperPath group reached significance on postoperative day 
Table 4 Preoperative and postoperative range of motion $\left(^{\circ}\right)$ within 12 months postoperatively

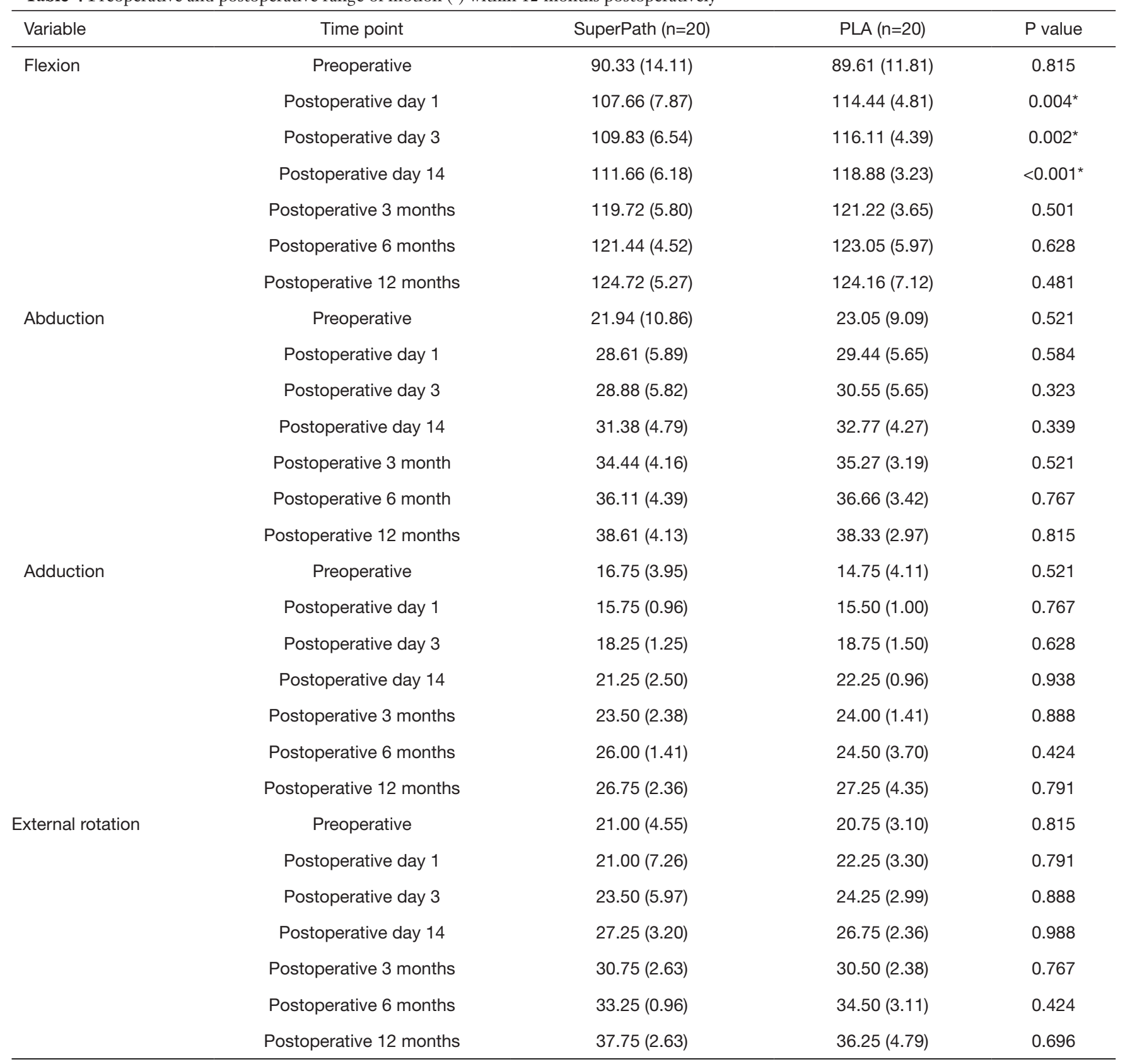

Data are reported as mean (standard deviation). Statistical analyses were performed with either Mann-Whitney U-test or $\chi^{2}$ test, where appropriate. *, P<0.05. PLA, posterolateral approach; SuperPath, supercapsular percutaneously-assisted total hip.

14. Similarly, hip function showed a significant improvement as early as postoperative day 14 in the PLA group, but not until 3 months postoperatively in the SuperPath group. These data indicated that SuperPath was associated with overall inferior patient-reported outcomes during the early postoperative phase in terms of pain symptoms and hip function. Moreover, cumulative evidence shows that patients with radiographically stable THA implants can develop chronic pain for unknown reasons (43). A recent nationwide survey in Denmark reported that $28 \%$ of THA patients suffered from chronic ipsilateral hip pain after 12 months postoperatively, of whom $12 \%$ had moderate to 

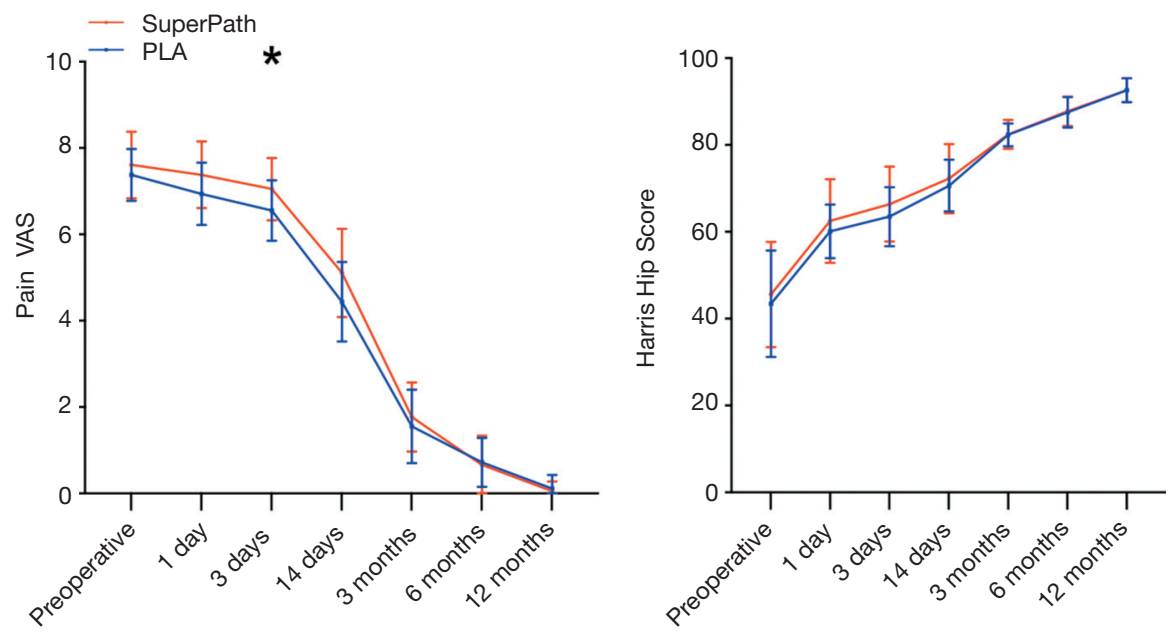

Figure 3 Perioperative changes in pain visual analog scale (VAS) and Harris Hip Score after unilateral total hip arthroplasty with either the supercapsular percutaneously-assisted total hip (SuperPath) or mini-incision posterolateral approach (PLA) for hip osteoarthritis. *, P<0.05 indicates statistical significance between the SuperPath group and PLA groups.

Table 5 Preoperative and postoperative pain VAS and HHS within 12 months postoperatively

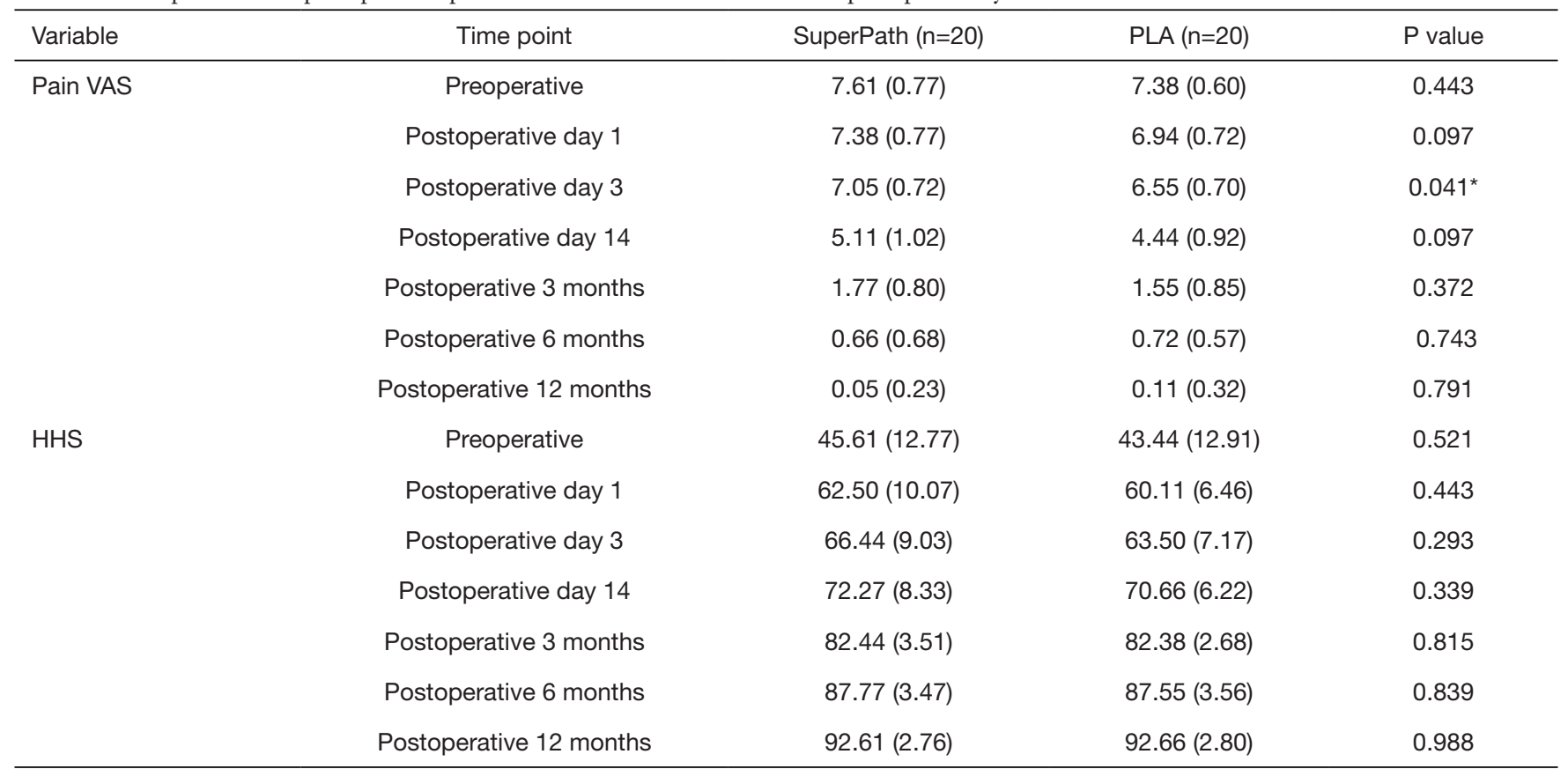

Data were reported as mean (standard deviation). Statistical analyses were performed with Mann-Whitney U-test or $\chi^{2}$ test, as appropriate. ${ }^{*}, \mathrm{P}<0.05$. VAS, visual analog score; HHS, Harris Hip Score; PLA, posterolateral approach; SuperPath, supercapsular percutaneouslyassisted total hip.

significant limitations on their daily activities due to chronic hip pain (44). Therefore, in the present study, which only had a 1-year follow-up, we were unable to determine the ultimate outcomes of the patients.
Acetabular component positioning has been recognized to be an essential variable in decreasing the risk of dislocation following either primary or revision THA (45). Unsatisfactory acetabular cup positioning might increase 
Table 6 Chronological changes of the pain VAS and HHS within 12 months postoperatively

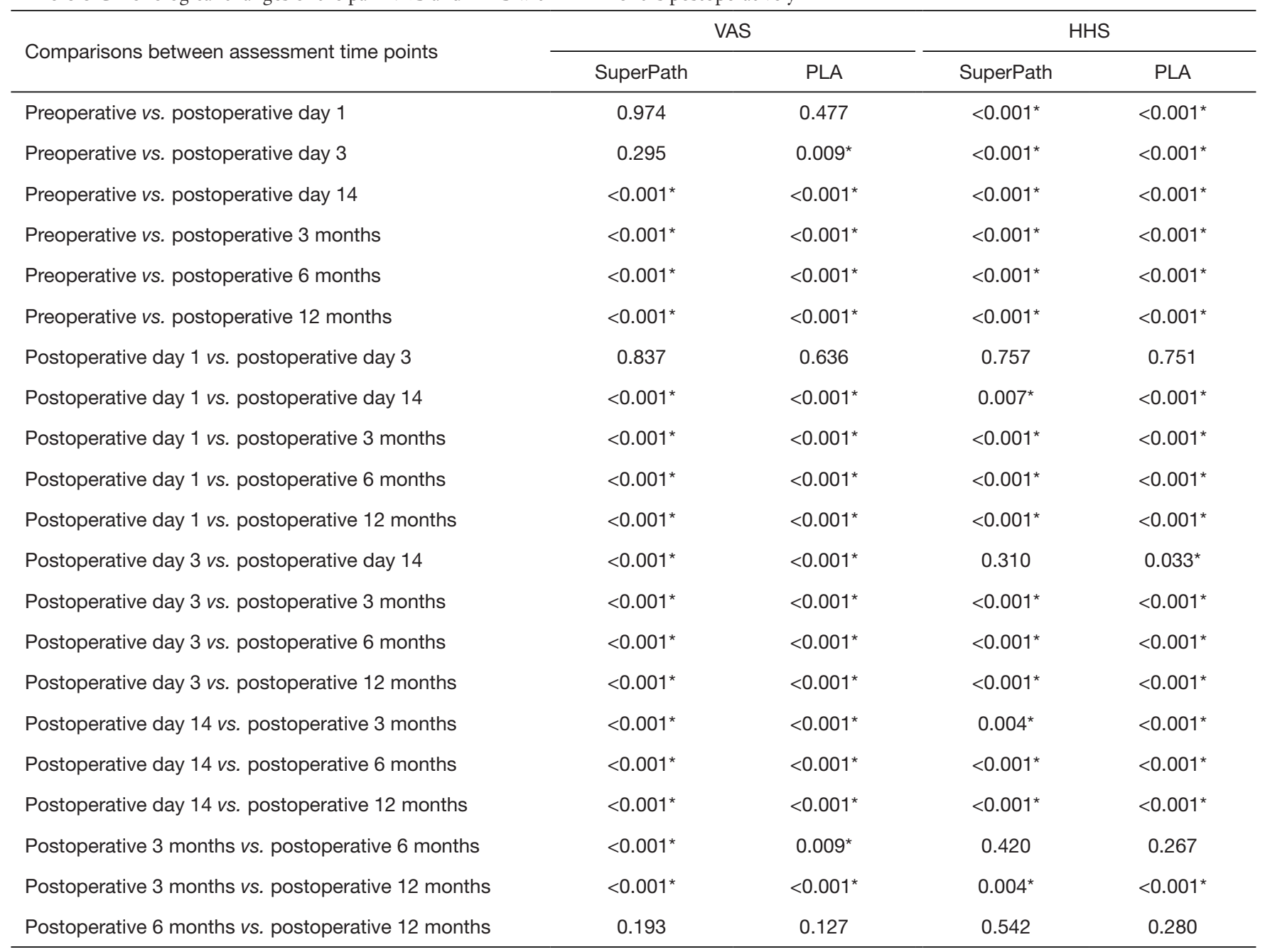

Comparisons of the pain VAS and HHS between each time point were analyzed with one-way analysis of variance using Tukey's honestly significant difference post-hoc test. *, P<0.05. VAS, visual analog score; HHS, Harris Hip Score; PLA, posterolateral approach; SuperPath, supercapsular percutaneously-assisted total hip.

the wear rate and deteriorate the long-term stability of the hip implants (46). We previously reported a relatively lower average abduction angle in the SuperPath group $\left(38.75^{\circ}\right)$ compared with the PLA group $\left(44.50^{\circ}\right)$ for bilateral osteonecrosis of the femoral head. Similarly, in the present study, we found a significantly lower average abduction angle in the SuperPath group compared with the PLA group for hip OA, which possibly serves as a cause for concern of cumulative long-term risk of dislocation. The more limited intraoperative exposure during the SuperPath procedure, which is an extra impediment to accurate manual positioning of the acetabular component, might have been responsible for these results. However, the impact of acetabular cup positioning on long-term outcomes of the SuperPath implant needs to be evaluated further.

SuperPath has recently attracted increased interest from orthopedic surgeons, with the possible advantages of tissue sparing and minimal invasiveness, for various indications of primary or revision THA, including femoral neck fracture and osteonecrosis of the femoral head $(16,32,47)$. However, to date, studies on SuperPath have been either cohort studies or case-control studies with $<2$-year follow-up $(21,35,47)$. This short-term follow-up does not allow for the determination of the ultimate outcomes of the SuperPath technique, considering that the implant survivorship in hip arthroplasty generally starts to diverge 
after 10 years postoperatively (48). However, a recent study on cost effectiveness reported that the SuperPath technique resulted in in-hospital cost reductions of over $28 \%$ compared with the standard lateral approach for THA (20). The present lack of long-term studies on SuperPath poses the question of whether this technique is cost effective. Currently available evidence is inadequate to either validate SuperPath as a superior alternative to conventional THA approaches or recommend its pathology-specific indications. More non-biased, high-powered, randomized controlled clinical trials to compare the long-term ultimate outcomes with in-depth cost-effectiveness analyses are necessary to determine the possible advantages of the SuperPath technique over conventional THA approaches, and to properly define its possible indications.

The present study has some limitations. First, the sample size was relatively limited without a power analysis performed in the research planning phase, and the postoperative follow-up was short. Second, the different hip implants utilized for both approaches might have influenced the postoperative outcomes. Third, the extent of muscle damage was only assessed with serum markers within 2 weeks postoperatively, while no radiographic analyses, such as magnetic resonance imaging, were performed to confirm perioperative alterations of these serum markers. Strengths of this investigation include its detailed and comprehensive chronological comparisons of robust patient-reported outcomes, radiographic parameters, and function scores for the first time between the SuperPath technique and conventional THA approaches for hip OA.

\section{Conclusions}

Although SuperPath is a minimally invasive technique, the present study shows, for the first time, that it had less favorable short-term outcomes than PLA for unilateral THA in patients with primary hip OA. Further studies are necessary to provide convincing evidence of SuperPath over other conventional mini-incision THA approaches.

\section{Acknowledgments}

The authors thank the anesthesiologists, nurses, and research pharmacy staff from the West China Hospital, Sichuan University for their support and collaboration.

Funding: Research funding and support was provided by the National Health and Family Planning Commission of the People's Republic of China (No. 201302007) and the Sichuan
Science and Technology Support Project (No. 2018SZ0145 and No. 2018SZYZF000). Weikun Meng received financial support from the China Scholarship Council.

\section{Footnote}

Reporting Checklist: The authors have completed the CONSORT reporting checklist. Available at http://dx.doi. org/10.21037/atm-20-1793a

Data Sharing Statement: Available at http://dx.doi. org/10.21037/atm-20-1793a

Peer Review File: Available at http://dx.doi.org/10.21037/ atm-20-1793a

Conflicts of Interest: All authors have completed the ICMJE uniform disclosure form (available at http://dx.doi. org/10.21037/atm-20-1793a). LG is a section editor of Annals of Translational Medicine. The other authors have no conflicts of interest to declare.

Ethical Statement: The authors are accountable for all aspects of the work in ensuring that questions related to the accuracy or integrity of any part of the work are appropriately investigated and resolved. The study was conducted according to the principles of the Declaration of Helsinki (as revised in 2013). The protocol of this study was approved by the Medical Ethics Committee of the West China Hospital, Sichuan University (No. 2016005). All patients provided signed consent for implanting and agreed to complete the scheduled postoperative 12-month follow-up.

Open Access Statement: This is an Open Access article distributed in accordance with the Creative Commons Attribution-NonCommercial-NoDerivs 4.0 International License (CC BY-NC-ND 4.0), which permits the noncommercial replication and distribution of the article with the strict proviso that no changes or edits are made and the original work is properly cited (including links to both the formal publication through the relevant DOI and the license). See: https://creativecommons.org/licenses/by-nc-nd/4.0/.

\section{References}

1. Quintana JM, Arostegui I, Escobar A, et al. Prevalence of knee and hip osteoarthritis and the appropriateness of joint replacement in an older population. Arch Intern Med 
2008;168:1576-84.

2. Gao L. Gene Therapy for Osteoarthritis Treatment and Joint Preservation. Int J Recent Surg Med Sci 2019;5:2-3

3. Kinsella K, He W. US Census Bureau, international population reports. Washington, DC: US Census Bureau, 2009.

4. Oláh T, Reinhard J, Gao L, et al. Topographic modeling of early human osteoarthritis in sheep. Sci Transl Med 2019;11:eaax6775.

5. Chang RW, Pellisier JM, Hazen GB. A cost-effectiveness analysis of total hip arthroplasty for osteoarthritis of the hip. JAMA 1996;275:858-65.

6. Schrenker S, Gao L, Cucchiarini M, et al. Future Aspects of Clinical Osteoarthritis Therapies in the Continuum of Translational Research. Z Orthop Unfall 2019;157:629-43.

7. Oláh T, Reinhard J, Gao L, et al. Reliable landmarks for precise topographical analyses of pathological structural changes of the ovine tibial plateau in 2-D and 3-D subspaces. Sci Rep 2018;8:75.

8. Gao L, Han Z, Xiong A. Total Hip Arthroplasty or Hemiarthroplasty for Hip Fracture. N Engl J Med 2020;382:1072-3.

9. Murphy BPD, Dowsey MM, Choong PFM. The Impact of Advanced Age on the Outcomes of Primary Total Hip and Knee Arthroplasty for Osteoarthritis: A Systematic Review. JBJS Rev 2018;6:e6.

10. Gao L, Madry H, Chugaev DV, et al. Advances in modern osteotomies around the knee : Report on the Association of Sports Traumatology, Arthroscopy, Orthopaedic surgery, Rehabilitation (ASTAOR) Moscow International Osteotomy Congress 2017. J Exp Orthop 2019;6:9.

11. Moore AT. The self-locking metal hip prosthesis. J Bone Joint Surg Am 1957;39-A:811-27.

12. Chechik O, Khashan M, Lador R, et al. Surgical approach and prosthesis fixation in hip arthroplasty world wide. Arch Orthop Trauma Surg 2013;133:1595-600.

13. Patel PD, Potts A, Froimson MI. The dislocating hip arthroplasty: prevention and treatment. J Arthroplasty 2007;22:86-90.

14. de Palma L, Procaccini R, Soccetti A, et al. Hospital cost of treating early dislocation following hip arthroplasty. Hip Int 2012;22:62-7.

15. Guo R, Gao L, Xu B. Current Evidence of Adult Stem Cells to Enhance Anterior Cruciate Ligament Treatment: A Systematic Review of Animal Trials. Arthroscopy 2018;34:331-340.e2.

16. Chow J, Penenberg B, Murphy S. Modified micro-superior percutaneously-assisted total hip: early experiences \& case reports. Curr Rev Musculoskelet Med 2011;4:146-50.

17. Murphy SB. Technique of tissue-preserving, minimallyinvasive total hip arthroplasty using a superior capsulotomy. Oper Tech Orthop 2004;14:94-101.

18. Penenberg BL, Bolling WS, Riley M. Percutaneously assisted total hip arthroplasty (PATH): a preliminary report. J Bone Joint Surg Am 2008;90 Suppl 4:209-20.

19. Chow J, Fitch DA. In-hospital costs for total hip replacement performed using the supercapsular percutaneously-assisted total hip replacement surgical technique. Int Orthop 2017;41:1119-23.

20. Gofton W, Fitch DA. In-hospital cost comparison between the standard lateral and supercapsular percutaneouslyassisted total hip surgical techniques for total hip replacement. Int Orthop 2016;40:481-5.

21. Meng W, Huang Z, Wang H, et al. Supercapsular percutaneously-assisted total hip (SuperPath) versus posterolateral total hip arthroplasty in bilateral osteonecrosis of the femoral head: a pilot clinical trial. BMC Musculoskelet Disord 2019;21:2.

22. Chow J. SuperPath: The Direct Superior Portal-Assisted Total Hip Approach. JBJS Essent Surg Tech 2017;7:e23.

23. Schaeffer JF, Scott DJ, Godin JA, et al. The Association of ASA Class on Total Knee and Total Hip Arthroplasty Readmission Rates in an Academic Hospital. J Arthroplasty 2015;30:723-7.

24. Xiao Q, Zhou Z. Perioperative pain management of total hip arthroplasty. Zhongguo Xiu Fu Chong Jian Wai Ke Za Zhi 2019;33:1190-5.

25. Li D, Yang Z, Kang P, et al. Home-Based Compared with Hospital-Based Rehabilitation Program for Patients Undergoing Total Knee Arthroplasty for Osteoarthritis: A Systematic Review and Meta-analysis of Randomized Controlled Trials. Am J Phys Med Rehabil 2017;96:440-7.

26. Poehling-Monaghan KL, Taunton MJ, Kamath AF, et al. No Correlation Between Serum Markers and Early Functional Outcome After Contemporary THA. Clin Orthop Relat Res 2017;475:452-62.

27. Widmer KH. A simplified method to determine acetabular cup anteversion from plain radiographs. J Arthroplasty 2004;19:387-90.

28. Huskisson EC. Measurement of pain. Lancet 1974;2:1127-31.

29. Lea RD, Gerhardt JJ. Range-of-motion measurements. J Bone Joint Surg Am 1995;77:784-98.

30. Harris WH. Traumatic arthritis of the hip after dislocation and acetabular fractures: treatment by mold arthroplasty. An end-result study using a new method of result 
evaluation. J Bone Joint Surg Am 1969;51:737-55.

31. Sculco TP. Minimally invasive total hip arthroplasty: in the affirmative. J Arthroplasty 2004;19:78-80.

32. Xu K, Anwaier D, He R, et al. Hidden blood loss after hip hemiarthroplasty using the superPATH approach: A retrospective study. Injury 2019;50:2282-6.

33. Cronin MD, Gofton W, Erwin L, et al. Early surgical and functional outcomes comparison of the supercapsular percutaneously-assisted total hip and traditional posterior surgical techniques for total hip arthroplasty: protocol for a randomized, controlled study. Ann Transl Med 2015;3:335.

34. Rasuli KJ, Gofton W. Percutaneously assisted total hip (PATH) and Supercapsular percutaneously assisted total hip (SuperPATH) arthroplasty: learning curves and early outcomes. Ann Transl Med 2015;3:179.

35. Xie J, Zhang H, Wang L, et al. Comparison of supercapsular percutaneously assisted approach total hip versus conventional posterior approach for total hip arthroplasty: a prospective, randomized controlled trial. J Orthop Surg Res 2017;12:138.

36. Tabatabai M, Segal R, Amidi M, et al. Serum creatine phosphokinase, lactic dehydrogenase, and their isoenzymes in the perioperative period. J Clin Anesth 1989;1:277-83.

37. Rykov K, Reininga IHF, Sietsma MS, et al. Posterolateral vs Direct Anterior Approach in Total Hip Arthroplasty (POLADA Trial): A Randomized Controlled Trial to Assess Differences in Serum Markers. J Arthroplasty 2017;32:3652-3658.e1.

38. Kumbhare D, Parkinson W, Dunlop B. Validity of serum creatine kinase as a measure of muscle injury produced by lumbar surgery. J Spinal Disord Tech 2008;21:49-54.

39. Capone A, Podda D, Civinini R, et al. The role of dedicated instrumentation in total hip arthroplasty. J Orthop Traumatol 2008;9:109-15.

Cite this article as: Meng W, Gao L, Huang Z, Wang H, Wang D, Luo Z, Bai Y, Wang G, Zhou Z. Supercapsular percutaneously-assisted total hip (SuperPath) versus miniincision posterolateral total hip arthroplasty for hip osteoarthritis: a prospective randomized controlled trial. Ann Transl Med 2021;9(5):392. doi: 10.21037/atm-20-1793a
40. Ogonda L, Wilson R, Archbold P, et al. A minimal-incision technique in total hip arthroplasty does not improve early postoperative outcomes. A prospective, randomized, controlled trial. J Bone Joint Surg Am 2005;87:701-10.

41. Mayer C, Franz A, Harmsen JF, et al. Soft-tissue damage during total knee arthroplasty: Focus on tourniquetinduced metabolic and ionic muscle impairment. J Orthop 2017;14:347-53.

42. Kwak S, Chun Y, Rhyu K, et al. Quantitative analysis of tissue injury after minimally invasive total hip arthroplasty. Clin Orthop Surg 2014;6:279-84.

43. Classen T, Zaps D, Landgraeber S, et al. Assessment and management of chronic pain in patients with stable total hip arthroplasty. Int Orthop 2013;37:1-7.

44. Nikolajsen L, Brandsborg B, Lucht U, et al. Chronic pain following total hip arthroplasty: a nationwide questionnaire study. Acta Anaesthesiol Scand 2006;50:495-500.

45. Sadhu A, Nam D, Coobs BR, et al. Acetabular Component Position and the Risk of Dislocation Following Primary and Revision Total Hip Arthroplasty: A Matched Cohort Analysis. J Arthroplasty 2017;32:987-91.

46. Hamilton WG, Parks NL, Huynh C. Comparison of Cup Alignment, Jump Distance, and Complications in Consecutive Series of Anterior Approach and Posterior Approach Total Hip Arthroplasty. J Arthroplasty 2015;30:1959-62.

47. Wang XD, Lan H, Hu ZX, et al. SuperPATH Minimally Invasive Approach to Total Hip Arthroplasty of Femoral Neck Fractures in the Elderly: Preliminary Clinical Results. Orthop Surg 2020;12:74-85.

48. Graw BP, Woolson ST, Huddleston HG, et al. Minimal incision surgery as a risk factor for early failure of total hip arthroplasty. Clin Orthop Relat Res 2010;468:2372-6.

(English Language Editors: R. Scott and J. Reynolds) 